\title{
Entre la geografía y la arqueología: el espacio como objeto y representación ${ }^{1}$
}

\author{
Emilio Alejandro Villafañez ${ }^{2}$
}

\begin{abstract}
RESUMEN
La geografía -ciencia del espacio- y la arqueología -encargada del estudio de las personas a través de sus restos materiales-, en el devenir de su historia han compartido mayor cantidad de temas de los que se suele suponer. La necesidad de examinar los grupos sociales y sus relaciones con el espacio es, en la actualidad, una de las claves para entender la dinámica de dichos grupos (tanto presentes como pasados). Si el espacio es un simple "telón de fondo" o una construcción social, en principio fue un problema solo de la geografía, aunque también influenció a otras ciencias, como el caso de la arqueología. En este artículo se brinda un panorama general a través de un análisis bibliográfico de las posturas teórico-metodológicas acerca de lo espacial, las que han aproximado a estas disciplinas.
\end{abstract}

Palabras clave: Geografía, arqueología, espacio, paisaje.

\begin{abstract}
Geography, the science of the spatial, and archeology, responsible for studying people through their material remains, have shared in the course of their history much more than is commonly assumed. The need to study social groups and their relationship to space is today, one of the keys to understanding the dynamics of these groups (both current and past). If space is a mere "backdrop" or a social construct, is something that at first was a problem only in geography, but which also influenced other sciences, as in the case of archeology. In this article, we provide a general panorama, analyzing existing bibliographic material, to review theoretical and methodological postures about what the spatial have meant to linking these disciplines.
\end{abstract}

Key words: Geography, archeology, space, landscape.

La intención de este trabajo es acercar a dos ciencias sociales con varias afinidades en el transcurso de su historia, y por diversas razones esta analogía -tanto a nivel teórico como metodológico- pocas veces se ha explicitado.

La geografía, como ciencia del espacio, fue una de las primeras en teorizar sobre

1 Artículo recibido el 21 de diciembre de 2010, aceptado el 5 de abril de 2011 y corregido el 17 de mayo de 2011. temas tales como la noción de "espacio" y "paisaje", y en cierto sentido se podría expresar que la arqueología fue la que tomó "prestados" dichos conceptos para su uso. Quisiera entonces caracterizar algunas correlaciones que existieron -y existen- entre ambas ciencias en relación al uso de esta terminología (Anschuetz et al., 2001; Curtoni, 2007; Segura, 2007).

\footnotetext{
2 Museo de Antropología, Universidad Nacional de Córdoba (Argentina). E-mail: emilio81@gmail.com
} 
La disposición es aclarar que este acercamiento mencionado anteriormente, se presenta de variadas formas, sin embargo, este escrito se centrará en los estudios respecto del espacio (y del paisaje), que estuvieron en sintonía con las revoluciones paradigmáticas devenidas en las ciencias sociales en general. Además, debo manifestar que no pretendo realizar una crítica acabada y completa al respecto, en cambio sí anhelo demostrar cómo en cierto punto estas ciencias se "tocaron" e intentaron un diálogo, de diversa índole, según el momento histórico y la región del mundo desde donde se escribe.

El trabajo se organizó en tres secciones. La primera (aunque bastante acotada) consiste en un acercamiento a la historia de las ciencias sociales, en referencia a cómo algunos paradigmas de moda permitieron a ciertas disciplinas gozar de una mayor relación entre sí. La segunda sección, vinculada con la primera, comprende específicamente, los inicios de la geografía y la arqueología en el marco de los paradigmas reinantes a lo largo del siglo XX, y cómo han utilizado al "espacio" y al "paisaje" como unidades de análisis. En la tercera parte se pretende analizar las aproximaciones y distanciamientos en torno de estos conceptos entre las perspectivas de algunos autores y textos que han mostrado una preocupación por poner en diálogo a estas disciplinas, intentando demostrar cómo la arqueología anglosajona tiene un vínculo estrecho aún con la geografía, suceso evidenciado de forma clara en sus trabajos, concepto no aplicable (del todo) a la arqueología latinoamericana.

\section{A modo de introducción}

A través de la historia de las ciencias sociales se puede observar como algunos paradigmas de moda establecieron que muchas disciplinas, ya formadas y otras en formación desde mediados del siglo XIX, tendieran a homogeneizarse en torno a ciertas formas de ver e interpretar el mundo.

Las influencias más importantes para la geografía y la arqueología, así como para las ciencias sociales en general, fueron las escuelas positivista y neopositivista hacia mediados del siglo XIX y principios del XX.
El positivismo tuvo como principales impulsores a Auguste Comte y John Stuart Mill, quienes postulaban que la observación es la base de todo conocimiento. $Y$ aquello factible de conocerse son las relaciones o conexiones entre los fenómenos estudiados, no la esencia de las cosas. La neutralidad y la objetividad son aspiraciones o pretensiones centrales del positivismo. El conocimiento de los vínculos debe llevar a la formulación de leyes que den cuenta de las nexos constantes existentes entre los sucesos examinados. Las leyes así establecidas tienen como fin la previsión racional, y puesto que ellas expresan un orden constante y necesario de dichos fenómenos observados, permiten prever el comportamiento futuro. El establecimiento de leyes, por otra parte, no implica valoración. Los juicios de valor, desde esta perspectiva, no tienen función cognitiva y deben ser evitados.

El neopositivismo o empirismo lógico (representado por la escuela de Viena) intentó ofrecer una visión general de la ciencia, la cual abarcaba principalmente sus aspectos gnoseológicos y metodológicos. Tal vez su tesis más conocida es aquella que sostiene que un enunciado es cognitivamente significativo solo si posee un método de verificación empírica o es analítico, postulado conocido como "del significado por verificación". Solamente los enunciados de la ciencia empírica cumplen con el primer requisito, y solo los de la lógica y las matemáticas cumplen con el segundo.

En este contexto nacen las denominadas nueva geografía y nueva arqueología, según las cuales, atentas al pretendido monismo metodológico, la explicación científica debía tener la misma forma en cualquier ciencia, específicamente a partir del ejemplo del método de estudio de las ciencias físico-naturales. El reduccionismo entonces se había apoderado de gran parte de las ciencias sociales, para las cuales la finalidad estaba puesta en descubrir generalizaciones y formular leyes de comportamiento, siendo la teoría general de sistemas, en algunos casos, un medio para poder lograrlo (Bertalanffy, 1976).

A partir de la década de 1960 comienza en el mundo occidental un período de profunda crisis donde se cuestionarán muchas de las ideas imperantes del modelo anterior, 
consideradas hasta entonces como inamovibles. En Estados Unidos se viven dos eventos políticos claves en los años 70: un movimiento por los derechos civiles frente a las desigualdades producidas por el sistema capitalista de producción y un fuerte repudio a la guerra de Vietnam y las pretensiones imperialistas del capitalismo dominante. En Europa sobresale el "mayo francés" de 1968, lo cual posibilita, junto a los mencionados movimientos, poner en jaque varias ideas establecidas hasta el momento acerca del estatuto de las ciencias sociales y del quehacer científico (Bertrand, 1987; Buzai, 2005).

Todos estos movimientos radicales convergen en una actitud antipositivista, cuya base ideológica germina en torno a la escuela de Frankfurt, una institución preocupada por profundizar en las teorías marxistas y también por encontrar soluciones a la postura del investigador respecto a las teorías y a la praxis. La teoría crítica de dicha escuela, desarrollada por Max Horkheimer, propone integrar la teoría y la acción y establece que el científico y la ciencia no son "neutrales" sino que forman parte de unas coordenadas socioeconómicas que los condicionan. También se propugnan enfoques más humanos para intentar comprender el conjunto de las ciencias sociales ya que, siendo el hombre el principal protagonista de ellas, debía ser tenido en cuenta en toda su dimensión y ser considerado no solo como ser productivo sino también como persona en su totalidad (Bertrand, 1987).

Es dentro de este movimiento donde diversas ciencias sociales se asientan y surgen posturas y escuelas radicalmente diferentes a sus predecesoras. Fueron los nuevos postulados provenientes tanto de la teoría social como del postempirismo, los movilizadores para que muchas disciplinas adopten diferentes formas de ver el mundo, como la estructuralista, la fenomenología o relecturas del marxismo, neomarxismo y en particular la teoría crítica (Scribano, 1999).

Con una base crítica en un comienzo y una fuerte postura teórica, empiezan a circular ciertas tendencias que luego se afianzarían a nivel mundial. La geografía humanista y la arqueología postprocesual forman parte de esas corrientes.

\section{Dos ciencias. ¿Un espacio, un paisaje?}

En este apartado pretendo exponer, en parte, cómo en la segunda mitad del siglo $X X$ algunas ciencias (como la geografía y la arqueología) flexibilizaron sus límites disciplinares logrando eliminarlos casi totalmente. Esto puede apreciarse en el uso de ciertos términos y metodologías que ya no son propios de una sola ciencia y empiezan a migrar, adaptándose a otras (Reboratti, 2001). El caso particular de los términos de "espacio" y "paisaje", los cuales fueron usados por la geografía y luego pasaron a la arqueología, es un claro ejemplo de ello.

\section{La geografía y arqueología como ciencias positivas}

Si le preguntáramos a cualquier persona (ajena al quehacer científico que estamos tratando) qué es el "paisaje", nos respondería casi con certeza, palabras más, palabras menos, que es una extensión de la superficie de la tierra que abarcamos con nuestra mirada (Farinelli, 2009).

La noción de "paisaje" nació hacia el siglo XVII en las artes plásticas, a partir de la pintura que representaba las escenas de la vida cotidiana europea, luego se trasladó a la arquitectura y después a la jardinería inglesa (Curtoni, 2007; Andrews, 2009; López Silvestre, 2009; Renes, 2009). La idea de "paisaje" empieza a tomar fuerza desde el mismo momento en que la geografía se institucionaliza como ciencia a principios del siglo XIX, lo cual generó diversas escuelas y hasta hubo quienes se animaron a hablar de la geografía como la "ciencia del paisaje". Esta última idea servía, en parte, para poder diferenciarse de otras ciencias a las que también les interesaba estudiar la superficie terrestre, como la ecología (Nogué i Font, 1985).

Para la Escuela Francesa de Geografía Regional, instaurada por Vidal de la Blache en la primera década del siglo XX, el paisaje era el reflejo de un estilo particular de la organización espacial, y aunque para dicho investigador el "paisaje" no era fundamental, sí lo era como parte del análisis de la región, esto último, objeto de estudio de la geografía 
(García Álvarez, 2006). Lucien Febvre, quien se inspiró en la geografía de Vidal de la Blache $^{3}$, fue uno de los máximos exponentes de la Escuela de los Annales, quien centró su investigación en el "paisaje", considerándolo básico para entender las relaciones entre las sociedades humanas y el medio geográfico.

Dentro de esta corriente y con anterioridad al desarrollo de la Segunda Guerra Mundial, la síntesis regional se consideraba el trabajo cúlmine del geógrafo (Chicharro Fernández, 1987). En la década del 50 este modelo entró en crisis, pues la realidad local que se describía ya no podía ser explicada por sí misma en un contexto dominado por la mundialización de las relaciones y, además, el esquema regional ya no ofrecía un marco adecuado para las políticas de planificación realizadas por los aparatos estatales.

Como explica Nogué i Font (1985), fue el rechazo de la nueva geografía hacia las posturas historicistas e ideográficas propias de la geografía regional, lo que llevó a la sustitución del término de "paisaje" por el de "espacio", concepto más adecuado a las exigencias positivistas y empiristas de este nuevo marco teórico.

3 Lucien Febvre y especialmente Marc Bloch (...) han seguido atentamente la historiografía social y económica alemana. (...). Mientras la mayor parte de los trabajos sobre la historia regional en Alemania se centraba, en aquella época (primer cuarto del siglo $X X)$, en la administración y en la constitución, lo que les interesaba a Lamprecht y a Febvre era la estrecha unión de las estructuras sociales, económicas y políticas con los modos de pensar y comportarse dentro de una determinada región. A diferencia de Alemania, en Francia (...) la geografía era un elemento fijo de la agrégation, el examen que era prácticamente obligatorio a fin de poder optar a una ulterior carrera universitaria como historiador. Y la geografía, que fue desarrollada en Francia por Paul Vidal de la Blache hacia 1900 (quien en muchos aspectos siguió la tradición de la geografía alemana del siglo XIX, encabezada por Carl Ritter), era una disciplina que situaba el espacio geográfico en un marco histórico-cultural. Vidal de la Blache, tal como fue entendido también por Febvre, evitaba en su géographie humaine el determinismo geográfico de su contemporáneo Friedrich Ratzel en Alemania (Iggers, 1998: 50).
En los años 60 entonces, el estudio de la región fue sustituido por el de los modelos espaciales dentro de una concepción filosófica neopositivista, favorecida por la llegada al mundo anglosajón de intelectuales precedentes del Círculo de Viena ${ }^{4}$, produciendo una nueva forma de hacer geografía.

De este modo nace la nueva geografía basada en la cuantificación de datos, siendo el abordaje básicamente espacial y con la clave central en la relación hombre-medio y en la diferenciación de espacios en la superficie terrestre. La región, como objeto de estudio, no se consideraba ya una realidad objetiva, sino algo que el investigador construía y delimitaba acorde a sus objetivos y marco teórico. La construcción de modelos era una de las finalidades de esta nueva corriente, atendiendo a características espaciales generalizables y usando metodologías rigurosas como las de las ciencias duras (matemática y física), las cuales le ofrecían otro lenguaje y le permitían un acercamiento a otras ciencias brindándole, teóricamente, una mayor objetividad (Buzai, 2005). Así, "la geografía pasó de estudiar objetos tangibles representables en el mapa al estudio de un objeto mucho más abstracto: el espacio (...) es así que, los nuevos geógrafos se concibieron a sí mismos como los protagonistas de una nueva revolución científica donde la ciencia del espacio se liberaba de su tradicional aislacionismo académico y se consideraba capaz de producir leyes y de generar una nueva literatura acumulativa por oposición a la anterior, de carácter aditivo" (Barros y Nastry, 1995: 11).

Si bien la geografía recibió aportes de otras ciencias, como la economía, no dejó de influenciar a su vez a otras disciplinas, como la arqueología.

\footnotetext{
4 Los miembros del Círculo de Viena publicaron en 1929 su manifiesto programático, titulado la visión científica del mundo. Propusieron utilizar un lenguaje común que debía ser elaborado por la filosofía, basándose en el lenguaje de la física, por ser esta la disciplina científica de mayores avances y la que practicaban profesionalmente muchos de los miembros del Círculo. Para el Círculo de Viena, la filosofía tiene la acepción de una disciplina más bien ligada a la lógica y el empirismo inglés, que define lo relevante de los enunciados.
} 
La arqueología puede ser definida como la ciencia social encargada de estudiar a las sociedades a través de sus restos materiales. Podría señalarse, además, que se caracteriza porque carece de información directa sobre el comportamiento humano, su objeto de estudio (Hernando, 1992).

Aunque con esta evidente "limitación", la arqueología científica de mediados del siglo XIX empezó a interpretar el pasado en base a los postulados evolucionistas que quedaban "confirmados" por los objetos obtenidos en el campo. Así, tomó suma importancia el modelo de Thomson, según el cual las sociedades habrían pasado por tres edades caracterizadas por el tipo de tecnología según el material empleado para su realización: edad de piedra, de bronce y de hierro.

Luego de este período, en las primeras décadas de 1900, tomó importancia el paradigma difusionista, basado en la migración como factor explicativo, lo cual dio inicio a una proliferación de teorías acerca de los círculos culturales, denominada históricocultural $^{5}$, que pretendía la identificación de grupos humanos. Esta nueva corriente dio nacimiento a los mapas de distribución de restos prehistóricos, con el fin de delimitar y seguir el desarrollo de sociedades específicas, lo cual habría servido a muchas naciones en plena formación de sus propios estados a fortalecer su identidad (Renfrew y Bhan, 2007).

En los años 40, el neoevolucionismo se apoderó de la antropología americana y fue Leslie White (uno de sus máximos exponentes) quien definió la cultura como un "medio extrasomático de adaptación". Luego uno de sus discípulos, Lewis Binford, adopta esas posturas y las inserta en la arqueología, dando lugar al origen de lo que Caldwell Ilamó "la nueva arqueología americana", dependiente casi por completo de la antropología

\footnotetext{
5 Uno de los padres del difusionismo europeo fue el geógrafo alemán Friedrich Ratzel, quien consideraba que todos los inventos se habían extendido por el mundo desde centros nucleares por medio de migraciones. Definió las áreas nucleares de difusión con el término alemán Kulturkreise (círculos culturales), y configuró un marco teórico de gran influencia en arqueología.
}

de los EE.UU. (Hernando, 1992). Binford esquematizó el programa de la nueva arqueología en dos artículos claves: "Archaeology as anthopology" (Binford, 1962), y "Archaeological systematics and the study of culture process" (Binford, 1965). Al considerar la cultura como un medio de adaptación al ambiente, dicho autor sostenía que el cambio no se daba por contacto con otras culturas, sino por influencia del ambiente, lo que llevó a defender la existencia de procesos generales de cambio, leyes que permitían entender el comportamiento humano, acercando el análisis arqueológico al de las ciencias naturales (Hernando, 1992), como sucedía en el caso de la nueva geografía y otras ciencias sociales.

Esta posición trajo aparejada una perspectiva positivista para tratar de entender los procesos socioculturales con un enfoque que se pretendía más objetivo. Esta revolución cientificista dio cabida a aproximaciones más empiristas, centrando la visión en las dimensiones físicas y visibles del hombre y su acción sobre el espacio. En estos momentos convergen tanto las perspectivas ecológico-cultural y funcionalista, la tradición de la "field archaeology" británica de los años 50, como los estudios cuantitativos característicos de la nueva geografía (Diez Martín, 2007).

La ruptura que significó el paradigma de la arqueología procesual surgió con una eminente vocación cientificista y racionalista, amparada en los enunciados del neopositivismo lógico propuestos por el filósofo alemán Carl Hempel. Según esta perspectiva, la recopilación de datos empíricos (observaciones arqueológicas) serviría para establecer leyes hipotéticas generales de carácter explicativo, independientes de las consideraciones históricas (Binford y Binford, 1968: 5-32). Los seguidores de esta arqueología científica se apoyaban firmemente en los principios de la ecología cultural o ecología humana, inicialmente propuesta por Steward y retomada posteriormente, entre otros, por Karl Butzer (1989).

A través de la aplicación de los principios de la teoría general de sistemas al campo de las ciencias sociales, los seguidores de la nueva arqueología asumen que el sistema cultural está compuesto por dos subsistemas 
básicos: el puramente sociocultural y el ambiental, los cuales interactúan intensamente en el desarrollo y evolución de las culturas. De este modo, el estudio del espacio como parte fundamental del subsistema ambiental, se convierte en un objetivo preferente. La variabilidad en la estructura territorial, pues, constituye una respuesta funcional (minimización de costes/optimización de la eficacia en la obtención de recursos) destinada a procurar estabilidad frente a los desafíos del cambio.

Los arqueólogos, influidos por las nuevas corrientes cuantitativas de la geografía británica (Orejas, 1991), dejarán de lado los estudios puramente descriptivos de corte espacial y utilizarán toda una gama de recursos metodológicos, matemáticos y estadísticos, destinados a medir y calibrar objetivamente los procesos arqueológicos en estudio. Es en el marco de la nueva arqueología y de todos sus principios teórico-metodológicos donde se asientan tanto el modelo del análisis de captación de recursos $(A C R)^{6}$ como las propuestas incluidas bajo el epígrafe de la arqueología espacial (De Carlos Izquierdo, 1990: 87). Tal posición llegó a tomar forma a partir de la publicación de los trabajos de Clarke (1977) y Hodder y Orton (1990). En líneas generales se puede definir la arqueología espacial como la "recuperación de información relativa a las relaciones espaciales arqueológicas y estudio de las consecuencias espaciales de las pautas de actividad homínida del pasado dentro y entre los contextos y estructuras, así como su articulación dentro de asentamientos, sistemas de asentamientos y sus entornos naturales" (Clarke, 1977: 47).

El centro de atención fundamental de la arqueología espacial hace referencia a las relaciones hombre-hombre (frente a las de hombre-medio del ACR), sea cual sea la escala de análisis abordada. A pesar de que en los estudios de corte espacial se ha reconocido un variado conjunto de aproximaciones, "...la arqueología del asentamiento, el análisis de los sistemas de yacimientos, los estudios regionales, el análisis territorial, el

\footnotetext{
6 Esta metodología nace, en el seno de la colaboración entre arqueólogos y geógrafos en la Universidad de Cambridge (Diez Martín 2007, entre otros).
}

análisis locacional, estudios de áreas de captación, los mapas de distribución, estudios de densidad, análisis intrayacimiento o de estructuras..." (Clarke, 1977: 89), se ha señalado que la mayor parte de estas parcelas de investigación podrían acomodarse en el marco del análisis locacional establecido en trabajos como los de Haggett, 1976 (De Carlos Izquierdo, 1992). Entre las aplicaciones de esta perspectiva podemos citar el modelo de vecino más cercano, los polígonos Thiessen, la teoría de lugar central y los modelos de Christaller, el modelo X-Tent o los límites y fronteras culturales (Hodder y Orton, 1990). Todos ellos parten de la extrapolación al campo arqueológico de procedimientos geográficos utilizados en el análisis de sociedades capitalistas $y$, por lo tanto, solo pueden aplicarse (no sin dificultades y críticas) en momentos con pautas estables de jerarquización espacial y especialización económica, esto es, en sociedades preferentemente productoras y complejas.

Por su parte, Renfrew critica los usos que se han hecho de estos modelos de Haggett: "El enormemente estimulante libro de $\mathrm{Ha}$ ggett sugiere muchos caminos en los que los arqueólogos pueden enfocar y analizar distribuciones en el espacio de materiales arqueológicos (tanto artefactos como de asentamientos o grupos culturales). No es una exageración decir que hemos estado trabajando sobre problemas que ya estaban resueltos" (Barros y Nastry, 1995: 12).

Un caso bastante distinto y merecedor de una particular atención, dentro de los estudios macroespaciales y de las corrientes procesuales, es el de la línea off-site o arqueología distribucional (Dunnell \& Dancey, 1983; Ebert, 1992; Foley, 1981). Los rasgos fundamentales que unifican este tipo de aproximaciones son los siguientes: 1) un enfoque eminentemente regional, destinado a esclarecer la estructura arqueológica a escala macroespacial y las interconexiones producidas entre los vestigios documentados; 2) un uso predominante del registro arqueológico reconocido en las superficies contemporáneas, sin por ello dejar de lado u obviar las secuencias estratigráficas disponibles.

A pesar de este último rasgo, la arqueología distribucional no debe entenderse como 
una mera estrategia de prospección ${ }^{7}$, sino como una metodología consistente de reconocimiento espacial y territorial. El artefacto, no el asentamiento, constituye la unidad básica de análisis, lo cual subraya la dimensión continuada del espacio arqueológico, su valor unitario $y$, por consiguiente, el hecho de que las altas acumulaciones de artefactos (los yacimientos tradicionales) solo suponen anomalías en la uniformidad territorial. La distribución de artefactos en el paisaje y la posterior interpretación se evalúan a través del parámetro densidad, esto es, las relaciones concentración/dispersión y presencia/ ausencia de objetos arqueológicos en el espacio regional. Las pautas de desecho a lo largo del paisaje se entienden como un proceso acumulativo a lo largo de un tiempo determinado que indican los comportamientos de estructuración del espacio y de recurrencia de determinadas actividades (áreas preferenciales y áreas complementarias de actividad); atención destacada a los factores postdeposicionales del registro que pueden haber condicionado o determinado las pautas de acumulación o dispersión observadas y, por ende, las inferencias establecidas (Belardi, 2005).

El concepto de espacio comienza a utilizarse de manera clara en este momento, pues en una primera etapa no había sido objeto de una discusión teórica fuerte, lo que implicó su uso acorde al sentido común propio de la sociedad occidental. Algunos autores como Clarke y Renfrew, exponentes de la nueva arqueología, fueron seducidos por el enfoque de la nueva geografía (aunque también críticos del uso exacerbado de algunas de sus metodologías) y su larga trayectoria en la utilización del término espacio, tomándolo como unidad de trabajo (Barros y Nastry, 1995).

Como se mencionó anteriormente, el interés por el espacio surgió a partir de los aportes de la nueva geografía que, influenciada por algunas teorías económicas, empezó

\footnotetext{
7 La prospección puede ser definida como "la aplicación de un conjunto de técnicas para optimizar la probabilidad de descubrimiento de los materiales culturales que caracterizan el registro arqueológico en el ámbito de un espacio geográfico conceptualmente definido" (Gallardo y Cornejo, 1985: 410).
}

a explicar la organización espacial de las sociedades basándose en el supuesto de que un grupo siempre tiende a maximizar las utilidades y a minimizar el esfuerzo-distancia. Estas posturas, tomadas por la nueva arqueología, permitieron que los sitios arqueológicos comenzaran a confeccionarse, verse e interpretarse como algo más allá que puntos en un mapa, guiados por ciertos métodos operativos.

\section{La crítica radical y las posturas humanistas}

Las críticas a los postulados extremadamente cientificistas llegaron a la geografía -como a todas las ciencias sociales- en los 70, considerando que hubo mayor preocupación por las técnicas analíticas que por el desarrollo y profundización de una auténtica teoría geográfica; además, el centro estuvo puesto en aspectos formales y concretos tomando distancia de los problemas relevantes; finalmente, se les acusa de caer en el Ilamado fetichismo espacial, convirtiendo el espacio en un objeto geométrico, teórico, separado de la vida concreta de la gente, justificador del orden social existente y carente de una dimensión ética.

Si la nueva geografía influenció a la nueva arqueología, fue la geografía humanista aqueIla disciplina que contribuyó a que el término "paisaje" tomara fuerza, tanto para su misma ciencia como para la arqueología postprocesual $^{8}$.

Fueron los convulsionados años 70 los cuales posibilitan el inicio de posturas más críticas en geografía. Entre estos casos se puede destacar en primera instancia a la geografía radical que nace con la aparición de la revista Antipode en 1969 (Pillet Capdepón, 2004), la cual advertía que esta ciencia, hasta ese

8 En sus inicios como investigador, lan Hodder estuvo muy comprometido con la arqueología espacial. Luego de este período marcadamente cientificista cambia su postura rotundamente, viendo la imposibilidad de justificar y asumir una racionalidad económica moderna en la toma de decisiones de los individuos o grupos sociales, en relación a los modelos y técnicas con que trabaja dicha arqueología. Años después dicho autor funda la arqueología postprocesual. 
momento, solo había servido para la guerra y para organizar mejor los "territorios" con objeto de controlar mejor a las sociedades. Con una base marxista y estructuralista, esta geografía logró poder acercarse a la sociología, preocupándose por las relaciones entre el espacio y el poder, y más concretamente por el espacio social, siendo sus máximos exponentes Milton Santos y David Harvey ${ }^{9}$.

Otro hecho significativo a tener en cuenta es la aparición de la geografía humanista. Con una base en los postulados de la fenomenología se declara anticientificista, porque a la sistematización del pensamiento humano opone el tomar en consideración otras verdades que no sean las demostradas por las leyes; es antipositivista porque considera que los comportamientos humanos no pueden ser estudiados según fórmulas matemáticas; y se considera antirreduccionista porque no cree que todas las actitudes humanas puedan ser relacionadas con algún lugar concreto del cerebro (Bertrand, 1987). Aunque no se niega que el hombre sea factible de ser estudiado desde un punto de vista científico, se estima que se deben tomar en consideración otras variables (Estébanez Álvarez, 2000).

Los humanistas tienen como postulado básico que el "espacio vivido es el mundo de la experiencia inmediatamente anterior al de las ideas científicas" (Sanguin, 1981: 561). Con este "espacio vivido" como punto de partida, definen en primera instancia el término de "lugar", que se refiere a "un área delimitada, a una porción del espacio concreto, caracterizada por una estructura interna distintiva y a la que se atribuye una significación que evoca siempre una respuesta afectiva" (Nogue i Font, 1985: 65); es en el "lugar" donde se encuentra un mundo de significados y sensaciones propias y ajenas.

Otro concepto clave hace referencia a

9 Estos autores volvieron a revalorizar al espacio como objeto de estudio en la geografía. Para Santos (1990; 2000), el espacio es un hecho social, un producto de la acción humana, una naturaleza socializada que puede explicarse básicamente por la producción. Harvey (1983), por su parte, veía al espacio como un producto social e intentó comprender su significado simbólico y sus complejas influencias en el comportamiento humano (Pillet Capdepón, 2004). la "experiencia", entendida como "la totalidad de nuestras relaciones con el mundo: sensaciones, percepciones, emociones, pensamientos" (Nogue i Font, 1985: 66). Fue Yi Fun Tuan, en su clásico libro denominado Topophilia (1974), donde examina las experiencias placenteras que ligan a los hombres con algunos lugares, donde la topofilia se contrapone a la topofobia ${ }^{10}$, la cual es sinónimo de aversión o miedo a determinados lugares. En este sentido, al autor afirma que el geógrafo humanista debe tener en cuenta los sentimientos, conceptos y teorías geográficas que posee el hombre y el grupo. Así, no debe despreciar el mundo de los hechos, sin embargo, debe tomar en consideración la significación hacia los mismos (Tuan, 1976).

De hecho, muchas de las reflexiones anteriores producen el resurgimiento del término de "paisaje" como forma de diferenciación del "espacio" de la nueva geografía (Nogue i Font, 1985). Basado en la fenomenología, el nuevo enfoque abre un amplio abanico para estudiar el paisaje como un fenómeno y constructo de nuestra propia actividad sensorial global, y no solo como algo dado o como un escenario ajeno a las percepciones del hombre.

Adentrándonos concretamente en el ámbito arqueológico, la corriente postprocesual, con una mirada también fenomenológica como base teórica, comenzó a realizar un análisis crítico en el que consideró que, si bien todas las estrategias propias de la nueva arqueología eran sumamente productivas y satisfactorias, basaban su rentabilidad en la ventaja del empirismo, consistente en que al definir y tratar con objetos reales, estos se pueden cuantificar, explicar, predecir, manipular. Sin embargo, el problema de estas estrategias consistía en el hecho de basarse en un empirismo reducido, en una noción simplificada de la realidad que no consideraba como entidades reales y objetos empíricos a las dimensiones no visibles. Lo que produjo el positivismo en la ciencia, desde esta nueva postura arqueológica, es que se ha olvidado especialmente de las dimensiones sociales y

\footnotetext{
10 También se pueden encontrar conceptos como topolatría, que refieren a los sentimientos reverenciales o míticos a ciertos lugares.
} 
culturales de los fenómenos que consideraba, de todas aquellas dimensiones que no tienen un carácter efectivo y físico concreto. Este tipo de elementos, al no ser controlables ni predecibles de modo directo, son más difíciles de manejar de forma eficaz e introducen indeterminación y márgenes de error en la investigación y en sus aplicaciones, razón por la que tradicionalmente se han dejado de lado (Thomas, 2001).

Esta visión más "abarcadora" de la ciencia arqueológica, la cual niega las posturas de un positivismo extremo, permitió el acercamiento de la disciplina en los componentes simbólicos del hombre y la manera en que crea, produce y reproduce el mundo en el que vive.

Saliendo de los límites de la perspectiva empirista que toma al paisaje como una realidad ya dada, se empieza a explicar el paisaje como el medio y a la vez el producto de los procesos sociales. Dentro de esta tendencia, Criado Boado (1999) propone concebir el paisaje como "el producto socio-cultural creado por la objetivación, sobre el medio y en términos espaciales, de la acción social tanto de carácter material como imaginario..." (Criado Boado, 1999: 5). Esta acción social está constituida tanto por las prácticas sociales como por la vida social misma.

A partir de esta conceptualización de paisaje se establece claramente que, a diferencia de otros seres vivos, los seres humanos no solo viven en el entorno, sino que crean su propio entorno para vivir o, dicho de otro modo, construyen su propio medio sociocultural. El estudio de este fenómeno desde el punto de vista arqueológico constituye, de hecho, la arqueología del paisaje (Criado Boado, 1995).

El paisaje, en cuanto producto social, se encuentra en realidad conformado por la conjunción de diferentes elementos, lo cual produce una nueva manera de ver el mundo que nos rodea:

- Un paisaje no es sinónimo de medio ambiente. Son los sistemas culturales quienes organizan y estructuran las interacciones entre la gente y el medio ambiente.

- El paisaje es una construcción cultural.
Son las comunidades quienes transforman los lugares físicos en espacios llenos de contenido.

- El paisaje, al formar parte de las actividades de una comunidad, no solo es un escenario construido por ellos, sino el lugar donde viven y se sustentan.

- Los paisajes son construcciones dinámicas en las cuales cada comunidad y generación imponen su propio mapa cognitivo, y establecen principios organizativos para la forma y estructura de cada sociedad (Anschuetz et al., 2001).

Así pues, una arqueología total del paisaje, en realidad se combina entre una "arqueología ambiental", una "arqueología del paisaje social" (parte de una arqueología social) y una "arqueología del paisaje imaginario" (perteneciente a una arqueología simbólica). Algunos de los problemas de diversas estrategias de análisis espacial y de un estudio arqueológico del paisaje, derivan precisamente del hecho de haberse centrado de manera exclusiva en una de esas orientaciones y haber elegido una sola de esas dimensiones como representación de la globalidad del paisaje (Criado Boado, 1999; Criado Boado, 1995).

Esta arqueología estudia al paisaje como un producto humano, quienes utilizan el espacio físico como una realidad dada, creando una nueva: el espacio humanizado, social, económico, mediante la aplicación de un orden imaginario, simbólico, sentido, percibido, pensado (Tilley, 1994).

\section{Encuentros y desencuentros entre la geografía y la arqueología}

El Ilamado "giro espacial", producido en estas últimas décadas en las ciencias sociales, ha desatado un acercamiento en estas ciencias, convirtiendo la transdisciplinariedad y la interdisciplinariedad en piezas claves entendidas como una "espacialidad explosiva" por Hiernaux y Lindón (2006). El giro cultural, por otra parte, permitió que muchas disciplinas y autores tomen los conceptos devenidos de la geografía, siendo uno de los casos más destacados los aportes de la geografía del tiempo de Hägerstrand tomados por Giddens (Lindón, 2006), quien ha efectuado uno de 
los desarrollos más importantes de la idea de espacio-tiempo en la teoría social contemporánea. Espacio-tiempo constituye un contexto dentro de los cuales son posibles, se sitúan, extienden y extinguen prácticamente todos los fenómenos sociales, con los cuales trabaja la teoría de la estructuración (Piazzini Suárez, 2006). A su vez, la geografía humanista encuentra todo su potencial en la teoría social, además de servirle como una forma de legitimarse como ciencia social (Hiernaux y Lindón, 2006).

Estimo que esta "espacialización" dada en las ciencias sociales influyó de manera profunda en arqueología, aunque a través de las lecturas hechas en geografía son escasas las menciones ${ }^{11}$ del impacto que tuvo -y aún sigue teniendo- la cuestión espacial, produciéndose un olvido -por parte de los geógrafos- de las influencias que tuvo en la arqueología.

En la bibliografía anglosajona se reconoce la inspiración fundamental que algunos enfoques geográficos, principalmente los de la geografía humanista, han ejercido en los estudios del paisaje en arqueología, permitiendo resaltar las formas en que los lugares se constituyen como espacios de significa-

\footnotetext{
11 Aunque no es el tópico de esta presentación, quiero destacar el caso de algunos geógrafos que se han preocupado por la arqueología y la necesidad de un trabajo interdisciplinario, por ejemplo Carls Sauers, quien se interesó por tener un diálogo constante con la arqueología y fue sin lugar a dudas, uno de los grandes innovadores en el estudio de la relación diacrónica y sincrónica entre el hombre y la tierra; estudio realizado a través de su propuesta sobre la geografía cultural, con conceptos y metodologías muy particulares, que son a la vez de una gran amplitud y constituyen parte inherente a este enfoque de frontera, caracterizado por ser en sí mismo interdisciplinario, al incluir aspectos básicos que articulan la naturaleza con la cultura. También se pueden rastrear algunos trabajos de David Harvey (ver Riley y Harvey, 2005). En el caso citado, estos autores se han preocupado por describir cómo la metodología de la historia oral puede relacionarse con los ámbitos del paisaje y los estudios de arqueología y patrimonio. Por último quiero resaltar también el caso del geógrafo argentino Carlos Reboratti quien ha tenido un inusitado interés hacia la arqueología, mostrando cómo el concepto de paisaje ha ido migrando de una ciencia a otra sin dejar relegada a la arqueología (para un ejemplo de ello ver Reboratti, S/D).
}

ción y el paisaje como un elemento activo y holístico (Tilley, 1994; Thomas, 2002). Además, y pese a no ser demasiado explicitado, muchas de las aproximaciones que conforman la arqueología del paisaje se apoyan en los estudios de geógrafos humanistas como Buttimer, Mugerauer, Pickles, Relph, Seamon y, sobre todo, Tuan, o en trabajos como los de Williams (2001) en un intento por otorgar mayor significación a los elementos arqueológicos que integran el paisaje. Es cierto que en ocasiones se ha generado una arqueología de los espacios vividos (Bender, 2002), sin embargo, y a diferencia de los estudios humanistas de los años 70 y 80 en los cuales la orientación predominante era lo particular (Tuan, 1974), el énfasis que algunos arqueólogos están incorporando en la última década ha apartado la búsqueda de imágenes individuales o de experiencias particulares, con el fin de ahondar en aproximaciones alejadas del modelo de paisaje eurocéntrico, y en las cuales el interés se centra tanto en las formas en que los paisajes son generados históricamente como en su interconexión con la construcción de identidades (Soler Segura, 2007).

Aunque junto a estas influencias, que en líneas generales pueden rastrearse en una amplia variedad de autores, existen otras que afectan de forma más individualizada. Deben destacarse, por la repercusión que tienen sus estudios dentro de la bibliografía especializada, las orientaciones explícitas que muestran determinados autores (Christopher Tilley desde la fenomenología o Julian Thomas con la hermenéutica), así como la influencia que en los estudios y reflexiones arqueológicas comienzan a tener las obras de sociólogos como Pierre Bourdieu, Anthony Giddens, o bien las de filósofos como Jacques Derrida, Michel Foucault, Jürgen Habermas, Martin Heidegger, Jean-François Lyotard, Paul Ricoeur, Richard Rorty o Gianni Vattimo. Esto parece evidenciar el reconocimiento, por una parte muy importante del colectivo investigador, de la necesidad de contar con un utillaje teórico y filosófico que permita superar las dificultades y límites que actualmente presenta el trabajo arqueológico. Pese a no ser una novedad esta instrumentalización de la teoría en arqueología (recordemos tan solo los préstamos de la nueva arqueología), parece que a diferencia de épocas pasadas, actualmente 
asistimos a una proliferación en el número de referentes teóricos empleados por los arqueólogos, los cuales indudablemente enriquecen la disciplina (Soler Segura, 2007).

En la literatura de habla hispana nos encontramos con algunos referentes a tener en cuenta, ante todo quisiera destacar el caso del arqueólogo español Vicent García (1991) que planteó un programa de investigación denominado "arqueo-geografía12", mediante el cual proponía, a través de los principios de la geografía agraria, estudiar la prehistoria de las sociedades campesinas del sureste de España. Con una fuerte base marxista, este autor señala que si bien los objetos son la base primaria con que trabaja el arqueólogo, son tan solo una parte de los medios de producción; esta restricción impide poder estudiar en su totalidad los problemas de las relaciones de producción, motivo por el cual toma a la tierra como un factor de producción determinante, y es allí donde entra en juego lo que él denomina "arqueología del paisaje agrario", cuyos fundamentos teóricos son

12 Por su parte, Javier de Carlos Izquierdo (1990) presentó una tesis denominada "La Arqueogeografía (un procedimiento para el estudio del espacio prehistórico)", en donde se exponía en su introducción de manera resumida, muchos de los temas planteados en el presente trabajo: "En aquellos momentos [1987, 1990], los arqueólogos nos afanábamos de leer la literatura geográfica con la finalidad de encontrar algún trabajo que pudiera adaptarse a nuestro objeto de estudio; a partir de ahí comenzó la reflexión que dio lugar a esta tesis. Durante aquella época una cierta sensación de frustración llegó a presidir la labor de los arqueólogos que se ocupaban de adaptar los avances de la geografía a la arqueología prehistórica. Fue entonces cuando comencé a asistir al seminario del Departamento de Geografía Humana de la Universidad Complutense, dirigido por el Dr. R. Méndez: la reflexión sobre los Procesos de Organización del Territorio debatidos en esas reuniones fue la verdadera piedra angular de este trabajo". La cita expuesta tiene más de autobiográfica que un aporte esencial para el trabajo; decidí agregarla aquí pues algo similar me ha sucedido. Cuando aún veía a la geografía solo como un manual de donde sacar nombres de ríos y montañas, participé en un curso de postgrado dictado por el geógrafo Carlos Reboratti, denominado "Conceptos transdisciplinarios: paisaje, ambiente, escala y territorio", y fue entonces cuando comprendí todos los aportes teóricos que la geografía ha brindado a las ciencias sociales, abriéndome un abanico de elementos teóricos y metodológicos que ahora considero fundamentales. los de la "geografía agraria" (Vicent García, 1991). De este modo, considera que "la geografía, en cuanto ciencia social del paisaje, tiene su propia dinámica teórica, paralela, en cierto modo, a la de la arqueología. Cuando los arqueólogos adoptamos un enfoque geográfico debemos tener esto en cuenta: nuestra percepción de nuestro espacio geográfico..." (Vicent García, 1991: 35).

El autor continúa expresando que al exponer el concepto de "arqueología del paisaje", establece una cierta oposición con la "arqueología medioambiental" vinculada a los paradigmas ecológico-culturales y evolucionistas. Debe entenderse que esta oposición sitúa a la "arqueología del paisaje" en el terreno de los problemas y métodos de la geografía, en cuanto ciencia social del espacio, sacándola del dominio teórico de la ecología. Esta afirmación lleva a plantear algunos problemas, siendo el más importante: ¿cómo puede verse este espacio social? Para esta pregunta sugiere dos posibles respuestas: la primera hace referencia a los estudios paleoclimáticos (estudios de polen) y la segunda a trabajos actualísticos (o sea la posibilidad de extrapolar los datos climáticos actuales al pasado). Vicent García esgrime que la prioridad práctica de las condiciones de observabilidad de los elementos del paisaje primitivo es solo aparente, y queda subordinada a un problema teórico de la geografía agraria, que no es otro que la propia elaboración conceptual de su objeto: el paisaje agrario. La evidencia paleoambiental posee en este enfoque un carácter de referencia de control más que de base de contrastación. Ahora bien, obviamente esta función es crucial. En consecuencia, la lectura arqueológica del paisaje agrario actual no es posible sin el desarrollo paralelo de una amplia estrategia de investigación paleoambiental y paleogeográfica.

La exposición anterior lleva a considerar al espacio agrario como resultado de la acción económica sobre el espacio natural, siendo sus componentes estructurantes una serie de elementos (agentes externos al paisaje, o sea aquellos rasgos observables que permiten describirlo) y factores (componentes explicativos de los elementos).

Para la descripción de estos elementos y factores, el arqueólogo toma una postura de 
la geografía prospectiva ${ }^{13}$, cuya finalidad es la explicación de la variabilidad paisajística, estableciendo para ello las relaciones determinantes entre la variabilidad de los factores y la morfología de los elementos.

La excusa para emplear esta metodología es que en la práctica, la localización de los asentamientos prehistóricos, con las limitaciones correspondientes a los niveles de información accesibles, es el único elemento fósil del paisaje agrario primitivo, en la medida en que pueda ser categorizado como una representación de la distribución del "hábitat rural" y utilizado en la descripción morfológica y funcional de los componentes del mismo (casas, agrupamientos, etc.). Esto nos obliga a construir toda nuestra metodología de acceso al paisaje primitivo a partir de este tipo de datos, lo cual explica que el análisis locacional sea el único campo de la geografía prospectiva que ha producido una cierta interacción metodológica con la arqueología. Estos modelos son, por ejemplo, los análisis de Von Tunen y Christaller, por mencionar algunos.

En resumen, la propuesta de Vicent García se inicia con un planteo que podría resultar obvio: la necesidad de estudiar el espacio siguiendo los principios de la ciencia que se encarga de estudiarlo, o sea, la geografía. Acercarse al paisaje agrario en base a los postulados de la geografía agraria es evidente, aunque tiene una postura marxista, para diversas explicaciones usa los modelos de la

\footnotetext{
13 Vicent García (1991:47) aclara que: El enfoque prospectivo en geografía se asocia normalmente a las corrientes neopositivistas - "Nueva Geografía"(Estébanez, 1984), señalándose igualmente sus implicaciones teóricas "neodeterministas" (Díaz Álvarez, loc. cit.). En el presente contexto, el término se debe tomar en un sentido más amplio, que denota, por oposición al "enfoque descriptivo", un objetivo explicativo y predictivo de los fenómenos geográficos, vinculado a categorías teóricas no necesariamente funcionalistas o evolucionistas, aunque sí materialistas. En este sentido, cabe distinguir el enfoque neopositivista de la geografía prospectiva, que tiene su inicio en el célebre artículo de F. Schaefer "Exceptionalism in Geography" (1953) -por tantos conceptos paralelos al "Archaeology as Anthropology" de Binford (1962)-, desenfoque prospectivo, en sentido genérico, que tiene su origen en la obra de J. H. von Thünen Der isolierte Staat in Bezeihung auf Landwirtschaft und Nationaloekonomie (1826 y 1850)".
}

geografía cuantitativa o nueva geografía, sin embargo, también aclara que "estos modelos deductivos no han sido concebidos, en principio, como una instancia explicativa, capaz de dar cuenta positivamente de los "patrones dé asentamiento", sino como un "dispositivo normativo", diseñado para resolver problemas concretos de decisión. Esto significa que no deben ser usados como explicaciones ad hoc de las configuraciones espaciales observadas, sino como un dispositivo de control, que permite aislar las desviaciones entre los patrones observados y los predichos por las hipótesis" (Vicent García, 1991: 48).

Otro autor importante es Emilio Piazzini Suárez, quien tiene una visión específica del tema espacial en arqueología desde Latinoamérica. El investigador nos explica que, en esta parte del mundo "las relaciones entre arqueología y espacio han sido abordadas, fundamentalmente, desde una perspectiva que enfatiza el tratamiento metodológico de este último (...) los análisis espaciales, incorporados y ajustados a partir de modelos desarrollados por la geografía y la ecología, son un acervo metodológico de la disciplina. No obstante el planteamiento de estas relaciones en el plano epistemológico y antológico no han sido frecuentes". Y continúa formulando que "esto parece relacionarse con una concepción implícita del espacio en su versión cartesiana, como extensión y soporte geofísico en el cual se desarrollan las prácticas y procesos sociales, y de la geografía y la ecología como saberes positivos sobre ese espacio y sus contenidos". Esta última cita remarca la clara falta de discusión teórica sobre el espacio, visto como un "telón de fondo", y la subordinación de este al tiempo, y cómo dichos términos fueron tomados por la arqueología como conceptos separados (y hasta opuestos en cierto sentido), con la modernidad se habían operado dos transformaciones: primero, el sentido de lugar se separó del espacio al incrementarse la relación entre ausentes, y segundo, espacio y tiempo se separaron en la medida en que tomó fuerza la regulación de las actividades sociales conforme a un tiempo homogéneo que no dependió de su localización (Piazzini Suárez, 2006).

Un cambio importante para el autor es el ya comentado giro espacial, lo cual permitiría tener en cuenta al menos tres observaciones. 
En primera instancia sería necesario partir de una consideración del espacio como sujeto, y del sujeto como algo especializado, tratando de construir un pensamiento del afuera, que surja de estimar que nuestra experiencia es forzosamente espacial. En segundo lugar se tendría que problematizar la oposición espacios objetivos y subjetivos, sin llegar a ningún extremo ya sea al tratar al espacio desde un punto de vista cartesiano o explicar su existencia desde visiones del todo fenomenológicas. En último lugar hay que intentar entender que la aparición del giro espacial no implica la muerte del tiempo, sino el ejercicio de repensar las relaciones entre espacio y tiempo.

Para finalizar, y para tratar de dar una solución a lo planteado, se pregunta ¿cómo ir más allá del discurso que predica la necesidad de utilizar paralelamente el tiempo y el espacio?, Piazinni Suárez no duda en citar a uno de los geógrafos más destacados en las últimas décadas, Milton Santos, quien propone emplear las categorías de espacio y tiempo según parámetros comparables; esto puede lograrse mediante una "empirización" del tiempo cuyo arraigo en el principio de sucesión, y no de simultaneidad (como ocurre con el espacio), lo concibe más abstracto. Tal "empirización" del tiempo sería posible al aproximarse a la materialidad de las técnicas como dato constitutivo del espacio y tiempo operacional y del espacio y tiempo constitutivos. "En este argumento se hace evidente la íntima conexión de las materialidades con el problema general del espacio-tiempo en una perspectiva que involucra directamente a la arqueología... En este sentido se puede esperar que, en principio, el estudio arqueológico de las materialidades pueda conducir a través de las interpretaciones de las relaciones y prácticas sociales en las cuales intervienen los artefactos, a la comprensión de experiencias específicas de espacio-tiempo" (Piazzini Suárez, 2006: 13).

Si quisiera seguir hablando de encuentros y desencuentros, podría escribir muchas líneas más de las aquí expuestas, pero solo he señalado algunas cuestiones fundamentales en relación al tema, que dan cuenta de las diversas formas de las arriba mencionadas: transdisciplinariedad y la interdisciplinariedad entre geografía y arqueología.

\section{Consideraciones finales}

En este trabajo resultó importante mostrar cómo dos ciencias sociales como la geografía y la arqueología han tenido un vínculo realmente mucho más estrecho de lo que diversas personas suponen. Aunque la vigencia y aporte mutuo entre la geografía y la arqueología anglosajona es evidente, no sucede lo mismo en esta parte del mundo. A pesar de los esfuerzos realizados por algunos investigadores para demostrarlo, considero que el devenir de la historia de la ciencia, a través del continuo paso de un paradigma a otro, ha hecho que dicha relación se vaya olvidando o, en el peor de los casos, que no haya resultado relevante.

Algunos investigadores afirman que la arqueología aún no ha logrado tener un método propio a nivel espacial, pero ¿es esto realmente necesario? Estimo que es válido en arqueología hacer uso de las teorías provenientes de la geografía, siempre y cuando se usen a conciencia, pues el desfase que existe entre ambas disciplinas es notorio.

En el primer cuarto de nuestro siglo, Barrow (1923) publicó "Geography as has human ecology", pero la arqueología no se incorporó totalmente a esta corriente antropológica hasta que tuvo lugar la segunda invectiva geográfica con la presentación de "Geography as human ecology", de Schnore (1961); en aquellos momentos Binford (1962) publicó "Archaeology as Anthropology". Del mismo modo la arqueología espacial de Clarke (1977) fue presentada académicamente un decenio después que Haggett (1965) publicara "Locational analysis in human geography". Esta misma tendencia ha continuado, y en 1987, Rowlands, Larsen y Kristiansen se ocuparon del modelo centro y periferia, que ya había sido propuesto a mediados de los 70 por Wallerstein (De Carlos Izquierdo, 1990: II).

La manera en que los estudios arqueológicos han fluctuado según las orientaciones que tomaba la geografía, es notorio, aunque según propia interpretación, es más clara la disociación (en algunas regiones) establecida entre la geografía y la arqueología (salvo pocos casos como ya se expuso). Si muchas 
otras ciencias (como la sociología y la antropología) supieron sacar provecho al "giro espacial" en las últimas décadas y les ha dado resultados más que prometedores, no encuentro motivo para que la arqueología no pudiera anhelar la misma suerte.

Actualmente existe un amplio consenso en considerar al espacio geográfico y arqueológico como un espacio social. Es entonces un producto de la acción humana, de allí que no sea una realidad dada ni preexistente, sino que se produce socialmente $y$, como tal, también históricamente.

"El espacio como producto social es un objeto complejo y polifacético: es lo que materialmente la sociedad crea y recrea, con una entidad física definida; es una representación social y es un proyecto en el que operan individuos, grupos sociales, instituciones, relaciones sociales, con sus propias representaciones y proyectos. El espacio se nos ofrece, además, a través de un discurso socialmente construido, que mediatiza al tiempo que vehicula nuestra representación y nuestras prácticas sociales. Es un producto social porque solo existe a través de la existencia y reproducción de la sociedad. Este espacio tiene una doble dimensión: es a la vez material y representación mental, objeto físico y objeto mental" (Ortega Valcárcel, 2004: 33-34).

La definición precedente es interesante por la riqueza y novedad de contenidos teóricos y porque permite presentar, de modo resumido aunque en sentido amplio, resultados y aportes de diversos autores dentro de las perspectivas actuales, tanto de geógrafos como de arqueólogos.

\section{Referencias bibliográficas}

ANDREWS, M. El paisaje en la poesía y la pintura románticas inglesas. En: MADERUELO, J. Paisaje e Historia. Madrid: Ed. Abada, 2009, p. 181-204.

ANSCHUETZ, K.; WILSHUSEN, R. \& SCHEICK, C. An archaeology of landscapes: perspectives and directions. Journal of archaeological research, vol. 9, $\mathrm{N}^{\circ} 2,2001$, p. 152-197.
BENDER, R. Time and Landscape. Current Anthropology, 2002, vol. 43, p. 103-112.

BARROS, C. y NASTRY, J. Estudio Preliminar. En: BARROS, C. y NASTRY, J. La perspectiva espacial en arqueología. Buenos Aires: Centro Editorial América Latina, 1995, p. 7-26.

BELARDI, J. Paisajes arqueológicos: un estudio comparativo de diferentes ambientes patagónicos. Oxford: BAR International Series 1390, 2005.

BERTALANFFY, L. Teoría general de sistemas. Buenos Aires: Ed. Fondo de Cultura Económico, 1976.

BINFORD, L. Archaeology asanthopology. San Diego: Ed. Academic Press, American Antiquity 11, 1962.

BINFORD, L. Archaeological systematics and the study of culture process. In: LEONE, M. (ed.). Contemporary Archaeology. Carbondale: University of Southern Illinois, 1965, p. 125-132.

BINFORD, S. \& BINFORD, L. Archaeological theory and method. In: SALLY, R. \& BINFORD, L. (eds.). New perspectives in archaeology. New York: Ed. Aldine, 1968, p. 1-3.

BERTRAND, C. El Sentido de la naturaleza: síntesis del pensamiento occidental y chino y su expresión en la pintura del paisaje. Tesis doctoral. Madrid: Universidad Complutense, 1987.

BUZAI, G. Geografía Cuantitativa 2000+. 20 lecciones fundamentales y sus tendencias de evolución. Revista de Geografía, 2005, vol. 5, No 1, p. 5-18.

BUTZER, K. Arqueología. Una ecología del hombre. Madrid: Ed. Bellaterra, 1989.

CURTONI, R. Arqueología y paisaje en el área centro-este de la provincia de La Pampa: la espacialidad humana y la formación de territorios. La Plata: Universidad Nacional de La Plata Facultad de Ciencias Naturales y Museo, 2007. 
CLARKE, D. Spatial archaeology. New York: Academic Press, 1977.

CRIADO BOADO, F. Construcción social del espacio y reconstrucción arqueológica del paisaje. En: BARROS, C. y NASTRY, J. La perspectiva espacial en arqueología. Buenos Aires: Centro Editorial América Latina, 1995, p. $75-116$

CRIADO BOADO, F. Del terreno al espacio: planteamientos y perspectivas para la arqueología del paisaje. Galicia: Ed. Grupo de investigación en arqueología del paisaje, Universidad Santiago de Compostela, 1999.

CHICHARRO FERNÁNDEZ, E. Notas sobre la evolución del pensamiento geográfico. Anales de Geografía de la Universidad Complutense, 1987, $\mathrm{N}^{\circ} 7$, p. 57-72.

DE CARLOS IZQUIERDO, J. La arqueogeografía (un procedimiento para el estudio del espacio prehistórico). Tesis Doctoral. Madrid: Universidad Nacional Complutense, 1990.

DIEZ MARTÍN, F. La arqueología del paisaje en la investigación paleolítica. Arqueoweb. Revista sobre arqueología en Internet, 2007, vol. 9, $\mathrm{N}^{\circ}$ 1. Disponible en Internet: http://www.ucm.es/info/arqueoweb/ pdf/9-2/diezmartin.pdf

DUNNELL, R. \& DANCEY, W. The siteless survey: A regional scale data collection strategy.. In: Schiffer, M. (ed.). Advances in Archaeological Method and Theory. New York: Academic Press, 1983, p. 267-287.

EBERT, J. Distributional Archaeology. Nuevo México: University of New Mexico Press, 1992.

ESTÉBANEZ ÁlVAREZ, J. Lecturas geográficas: homenaje a José Estébanez Álvarez. Madrid: Ed. Universidad Complutense, 2000.

FARINELLI, F. El don de Humboldt: el concepto de paisaje. En: COPETA, C. y LOIS, R. Geografía, paisaje e identidad. Madrid: Ed. Biblioteca nueva, 2009, p. 43-82.
FOLEY, R. A model of regional archaeological structure. Proceedings of the prehistoric society, 1981, No 47, p. 1-17.

GALLARDO, F. A. y CORNEJO, L. Definiendo el sitio arqueológico: metodología en acción. Arica: Universidad de Tarapacá, 1985.

GARCÍA ÁLVAREZ, X. Geografía regional. En: HIERNAUX, D. y LINDÓN, A. Tratado de Geografía Humana. Ciudad de México: Ed. Anthopos, 2006, p. 25-70.

HAGGETT, P. Locational análisis in human geography. London: Ed. Edward Arnold, 1965.

HERNANDO GONZALO, A. Enfoques teóricos en arqueología. Revista de prehistoria y arqueología, 1992, vol. 13, No 1, p. 11-35.

HODDER, I. y ORTON, C. Análisis espacial en arqueología. Barcelona: Crítica, 1990.

HIERNAUX, D. y LINDÓN, A. Introducción. La geografía humana: un camino a recorrer. En: HIERNAUX, D. y LINDÓN, A. Tratado de Geografía Humana. Ciudad de México: Ed. Anthopos, 2006, p. 7-22.

IGGERS, G. La ciencia histórica en el siglo XX. Barcelona: Idea Books S.A., 1998.

LINDÓN, A. Geografías de la vida cotidiana. En: HIERNAUX, D. y LINDÓN, A. Tratado de Geografía Humana. Ciudad de México: Ed. Anthopos, 2006, p. 356-400.

LÓPEZ SILVESTRE, F. Pensar la historia del paisaje. En: MADERUELO, J. Paisaje e Historia. Madrid: Dir. Ed. Abada, 2009, p. 9-52.

NOGUE I FONT, J. Geografía humanista y paisaje. Anales de geografía de la Universidad Complutense, $\mathrm{N}^{\circ}$ 5, 1985, p. 93-107.

OREJAS, A. Arqueología del paisaje: Historia, problemas y perspectivas. Archivo Español de Arqueología, № 64, 1991, p. 191230. 
ORTEGA VALCÁRCEL, X. Los horizontes de la geografía: teoría de la geografía. Barcelona: Ed. Ariel, 2004.

PIAZZINI SUÁREZ, E. Arqueología, espacio y tiempo: una mirada desde Latinoamérica. Arqueología Suramericana, 2006, vol. 2, No 1, p. 3-25.

PILLET CAPDEPÓN, F. La geografía y las distintas acepciones del espacio geográfico. Investigaciones geográficas, 2004, № 34, p. 141-154.

REBORATTI, C. Una cuestión de escala: sociedad, ambiente, tiempo y territorio. Sociologías, 3/5, 2001, № 3, p. 16-28.

REBORATTI, C. El paisaje como concepto migrante. Buenos Aires: inédito S/D.

RENES, J. Paisajes europeos: continuidades y transformaciones. En: MADERUELO, J. Paisaje e Historia. Madrid: Ed. Abada, 2009, p. 53-88.

RILEY, M. \& HARVEY, D. Landscape Archaeology, Heritage and the Community in Devon: An Oral History Approach. International Journal of Heritage Studies, vol. 11, No 4, p. 269-288, 2005.

SANGUIN, A. la géographie humanie uo I'approche phénomenologique des lieux, des paysages et des espaces. Annales de géographie, 1981, №501, p. 586-584.

SANTOS, M. Por una geografía nueva. Madrid: Ed. Espasa, 1990.

SANTOS, M. La naturaleza del espacio. Técnica y tiempo. Razón y emoción. Barcelona: Ed. Ariel GEOGRAFÍA, 2000.

SCRIBANO, A. Epistemología y teoría: un estudio sobre Bourdieu, Giddens y Habermas. Catamarca: Ed. Dirección General del Centro Editor, Secretaría de de Ciencia y Tecnología, Universidad Nacional de Catamarca, 1999.

SEGURA, A. Redefiniendo en registro material. Implicaciones recientes de la arqueología del paisaje anglosajona. Trabajos de prehistoria, 2007, vol. 64, № 1, p. 41-64.

SOLER SEGURA, J. Redefiniendo el registro material. Implicaciones recientes desde la arqueología del paisaje anglosajona. Trabajos de prehistoria, 2007, vol. 64, № 1 , p. 41-64.

TILLEY, C. A phenomenology of landscape: places,paths, and monuments. Oxford: Berg, 1994

THOMAS, J. Archaeologies of Place and Landscape. In: HODDER, I. (ed.). Archaeological Theory Today. Cambridge: Polity, 2001, p. 165-186.

TUAN, Y. Thopophilia: a study of environment perception, attitudes and values. New York: Prentice Hall, 1974.

TUAN, Y. Humanistic geography. Annals of Association of American Geographers, 1976, vol. 66, N² 2, p. 266-276.

RENFREW, C. y BHAN P. Arqueología. Teoría, métodos y práctica. Madrid: Akal ediciones, 2007.

VICENT GARCíA, J. Fundamentos Teórico-Metodológicos para un programa de investigación arqueo-geográfica. En: LÓPEZ GARCÍA, P. Cambio cultural del IV al II milenios a. C. en la comarca del Noroeste de Murcia. Volumen I. Madrid: Consejo Superior de Investigaciones científicas, 1991, p. 31117.

WILLIAMS, R. El campo y la ciudad. Barcelona: Paidós, 2001. 